\title{
A NOTE ON THE IDEAL [uv]
}

D. G. MEAD

If $P \in[u v]$ has signature ${ }^{1}\left(d_{1}, d_{2}\right)$ and weight $d_{1} d_{2}$, by Levi's reduction process $\left[1\right.$, p. 561] it is known that $P \equiv c_{1} u^{d_{1}} v_{d_{1}}^{d_{2}}$ for some number $c_{1 .}{ }^{2}$ If one were to use the symmetric reduction process (obtained by interchanging the roles of $u$ and $v$ ) he would find $P \equiv c_{2} u_{d_{2}}^{d_{1} d_{2}}$; hence

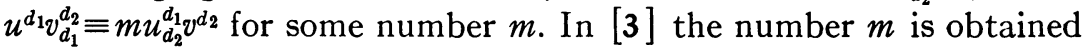
when $d_{1}=d_{2}$. The number $m$ for all positive $d_{1}$ and $d_{2}$ is given by the

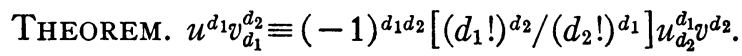

Lemma I(a). If $P$ involves both $u$ and $v$ and $P u_{i}$ and $P v_{j}$ have excess weights [2, p. 426] of 0 and 1 respectively, then:

$$
P u_{i} v_{j} \equiv(-j /(i+1)) P u_{i+1} v_{j-1} .
$$

ProOF.

$$
\begin{array}{r}
P u_{i} v_{j} \equiv-P\left[\left(\sum_{k=1}^{i} \frac{i ! j !}{(i-k) !(j+k) !} u_{i-k} v_{j+k}\right)+\frac{i ! j !}{(i+1) !(j-1) !} u_{i+1} v_{j-1}\right. \\
\left.+\sum_{k=2}^{j} \frac{i ! j !}{(i+k) !(j-k) !} u_{i+k} v_{j-k}\right] .
\end{array}
$$

The typical term in the first sum contains $P u_{i-k}, k \geqq 1$, which has negative excess weight and thus is in [uv]. Similarly, the typical term in the second sum contains $P v_{j-k}, k \geqq 2$, and, having negative excess weight, is in $[u v]$.

LEMMA I(b). If $Q$ is a pp. in $v$ alone and $Q u_{j} v_{1}$ is of excess weight 0 , then

$$
Q u_{j} v_{1} \equiv-\frac{1}{j+1} Q u_{j+1} v .
$$

The proof is so similar to the one just given that the details will be omitted.

Assume $d_{1}$ and $d_{2}$ are fixed positive integers. For $0 \leqq i<d_{2}, 0 \leqq s<d_{1}$ and for $i=d_{2}, s=0$ let

$$
R(i, s)=\stackrel{d_{i}-s}{d_{i}^{s}} u_{i+1}^{i} v^{i} v_{d_{1}-s} v_{d_{1}}^{d_{2}-(i+1)}
$$

Received by the editors May 8, 1962.

${ }^{1}$ Some familiarity with the nomenclature employed in [1] and [2] is assumed.

2 In this note all congruences are modulo $[u v]$. 
LEMMA 2.

$$
u^{d_{1} d_{d_{2}}} v_{d_{1}} \equiv(-1)^{i d_{1}+s} \frac{\left(d_{1} !\right)^{i+1}}{(i !)^{d_{1}-s}(i+1)^{s}\left(d_{1}-s\right) !} R(i, s) .
$$

Proof. The lemma is clearly true if $i=s=0$, and the proof is completed using induction on the pair $(i, s)$ ordered lexicographically. We assume the lemma valid for the pair $(i, s)$ and distinguish two cases.

First, if $0 \leqq s<d_{1}-1$, we need only show

$$
R(i, s) \equiv-\frac{d_{1}-s}{i+1} R(i, s+1) .
$$

By Lemma I(a) with $P=u_{i}^{d_{1}-s-1} v^{i}$ and $j=d_{1}-s$

$$
\begin{aligned}
R(i, s) & =u_{i}^{d_{1-s-1}} v^{i} u_{i} v_{d_{1-8}} u_{i+1}^{s} v^{d_{2}-(i+1)} \\
& \equiv-\frac{d_{1}-s}{i+1} u_{i}^{d_{1-s-1}} v^{i} u_{i+1} v_{d_{1-8-1}} u_{i+1}^{s} v_{d_{1}}^{d_{2}-(i+1)} \\
& =-\frac{d_{1}-s}{i+1} R(i, s+1) .
\end{aligned}
$$

Second, if $s=d_{1}-1$, by Lemma I(b) with $Q=v^{j}$ we see

$$
R\left(j, d_{1}-1\right)=v^{j} u_{j} v_{1} u_{j+1}^{d_{1}-1} v_{d_{1}}^{d_{2}-(j+1)} \equiv-\frac{1}{j+1} u_{j+1}^{d_{1} v^{j+1} v_{d_{1}}^{d_{2}-(j+1)}} .
$$

Combined with the induction assumption, this completes the proof of Lemma 2. The validity of the theorem, which is a special case of Lemma 2 (when $i=d_{2}, s=0$ ), has thus been demonstrated.

\section{BiBLIOGRAPHY}

1. H. Levi, On the structure of differential polynomials and on their theory of ideals, Trans. Amer. Math. Soc. 51 (1942), 532-568.

2. D. G. Mead, Differential ideals, Proc. Amer. Math. Soc. 6 (1955), 420-432.

3. K. B. O'Keefe, A symmetry theorem for the differential ideal [uv], Proc. Amer. Math. Soc. 12 (1961), 654-657.

Pratt Institute 